\title{
Évaluation Des Pratiques Phytosanitaires Paysannes Dans Les Vergers De Cacao Dans Le Departement De Daloa, Côte d'Ivoire
}

\author{
Siapo Yao Martin, \\ Tahiri Annick, \\ Ano Ehui Joachim,
}

Laboratoire d'Endocrinologie et de Biologie de Reproduction, UFR Biosciences, Université Felix Houphouët Boigny, Abidjan, Côte d'Ivoire

\section{Diby Yao Kan Séraphin,}

Laboratoire de Zoologie et Biologie Animale, UFR Biosciences, Université

Felix Houphouët Boigny, Abidjan, Côte d'Ivoire

Doi: 10.19044/esj.2018.v14n33p267 URL:http://dx.doi.org/10.19044/esj.2018.v14n33p267

\begin{abstract}
This paper focuses on identifying pesticides uses in the protection of cocoa tree. To do this, a survey was conducted among 129 randomly selected cocoa producers in the Daloa zone in Côte d'Ivoire. The results showed that $79.84 \%$ of the producers interviewed, use pesticides. Twenty-seven (27) pesticides were identified; among them, 24 were insecticides, 3 were fungicides, and two were not licensed. More than 50\% of the insecticides used by famers were neonicotinoids combined with pyrethroids. None of the pesticides was termiticide although termite damage was cited as major. Fiftythree point four percent $(53.4 \%)$ of producers store pesticides in their house. Empty packs and containers are abandoned in nature after being used by $96 \%$ of surveyed farmers, while $4 \%$ reused them. The results indicated that producers should be well sensitized on good agricultural practice.
\end{abstract}

Keywords: Pests, Cocoa producers, Phytosanitary practices, Côte d'Ivoire

Résumé

Cette étude a pour objectif d'identifier les pesticides et de connaître si leurs utilisations sont axées sur la protection du cacaoyer. Pour ce faire, une enquête a été menée auprès de 129 producteurs de cacao dans la zone de Daloa en Côte d'Ivoire. Il ressort de cette étude que 79,84\% des producteurs interrogés utilisent des pesticides. Vingt-sept pesticides, dont 24 insecticides et 3 fongicides, ont été recensés et dont deux ne sont pas homologués. Plus 
de $50 \%$ des insecticides sont des néonicotinoïdes combinés avec les pyréthrinoïdes. Parmi les pesticides, aucun n'est termiticide bien que les dégâts par des termites sont cités comme majeurs. Les résultats de l'étude montrent aussi que cinquante-trois virgule quatre pour cent des producteurs stockent les pesticides dans leur habitation à accès libre. Les emballages et les contenants vides sont abandonnés dans la nature après usage par $96 \%$ des enquêtés tandis que $4 \%$ les réutilisent. Autrement dit, les résultats indiquent que les producteurs devraient être sensibilisés aux bonnes pratiques agricoles.

Mots clés: Ravageurs, Producteur de Cacao, Pratiques phytosanitaires, Côte d'Ivoire

\section{Introduction}

La diversité climatique et écologique de la Côte d'Ivoire est certes favorable au développement des diverses productions agricoles. Cependant, elle est également propice à la prolifération des bioagresseurs qui nuisent aux cultures. Parmi ces cultures figure la cacaoculture, fortement attaquée par plusieurs ravageurs (Tra-Bi et al., 2010 ; Kouakou et al., 2011 ; N'Guessan et al., 2014 ; Kouamé et al., 2015).

Pour contrôler ces ravageurs, les pesticides chimiques sont de plus en plus utilisés par les producteurs, contribuant à une pratique agricole basée sur une utilisation importante d'intrants chimiques dans les cacaoyers en Côte d'Ivoire. L'utilisation de ces produits n'est pas sans effets néfastes sur l'homme et l'environnement (Hoyer et al., 2002 ; Ladjide et al., 1995). De même, la non maîtrise par les paysans des pratiques agricoles pourrait accentuer les intoxications et les pollutions. Pour cette raison, il nous a semblé nécessaire d'identifier les pesticides et les pratiques d'utilisations sur le cacaoyer dans une zone de production de cacao, le département de Daloa, au Centre - Ouest de la Côte d'Ivoire.

\section{Matériel et Méthodes \\ Zone d'étude}

L'étude a été réalisée dans des villages, à proximité de la ville de Daloa, au Centre-Ouest de la Côte d'Ivoire. La zone de Daloa est caractérisée par un climat chaud et humide de type tropical avec des précipitations comprises entre 1000 et $1500 \mathrm{~mm}$ par an (Brou et $a l$., 2005). Le sol est de type ferralitique dérivé de schistes quartzitiques riches en argile avec un bon pouvoir de rétention en eau. La température moyenne annuelle est de $25,6^{\circ} \mathrm{C}$ (Anonyme, 2018). 


\section{Méthodologie}

Cette enquête a été basée sur une étude transversale de type descriptive. Elle a été réalisée dans six villages producteurs de cacao à proximité de la ville de Daloa, encadrés par l'Agence Nationale de Développement Rural (ANADER) - Zone de Daloa. L'enquête a été semi-ouverte et déclarative. Au total, 129 producteurs de cacao ont été sélectionnés et interviewés individuellement. Le questionnaire, divisé en 3 parties, abordait: (1) des informations sur le producteur (l'âge, le sexe, le niveau d'instruction), puis (2) le verger (surface, âge, variété de cacaoyers), et enfin (3) les produits phytosanitaires (ravageurs, maladies, traitements chimiques, fréquences, doses/ha, cible, période, stockage et devenir des emballages vides).

\section{Résultats}

\section{Typologies des producteurs}

Près de $94,57 \%$ des producteurs interrogés sont des hommes. La majorité $(80,61 \%)$ des producteurs sont âgés de plus de 35 ans (Figure 1). La moyenne d'âges est de $45 \pm 11,81$ ans. Seulement $34,95 \%$ des producteurs sont analphabètes (Figure 2).

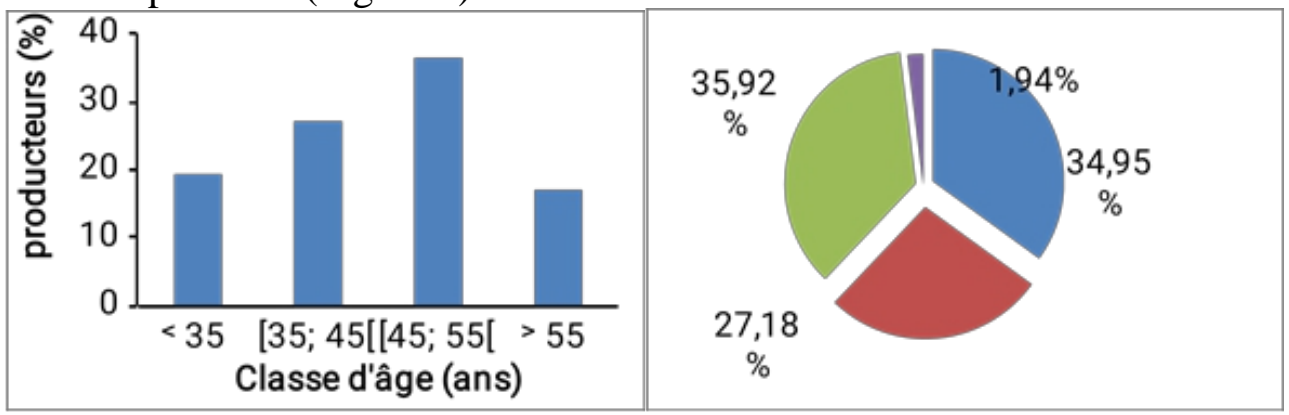

Figure 6. Classe d'âge des producteurs

Figure 7. Niveau d'instruction des producteurs

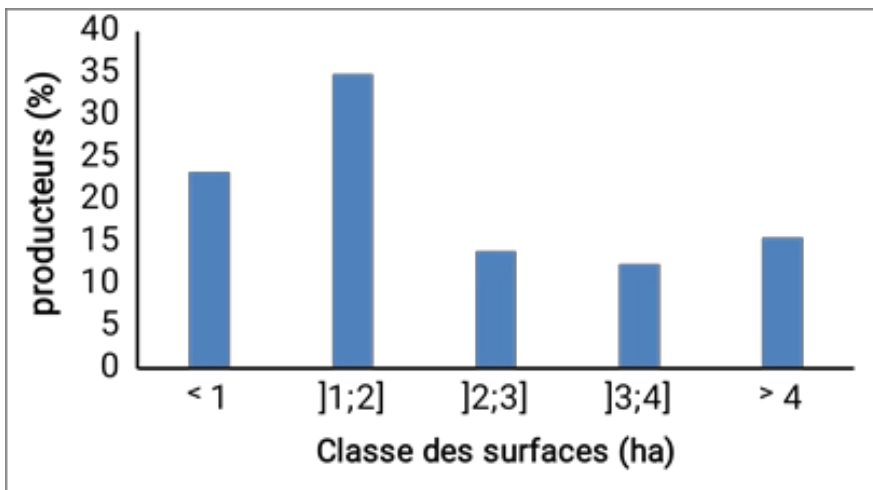

Figure 8. Classe des surfaces des plantations 
La taille moyenne des parcelles exploitées est de 2,62 $\pm 1,80$ ha. $\mathrm{La}$ majorité $(34,88 \%)$ des producteurs cultivent entre 1 et 2 ha, tandis que $23,26 \%$ cultivent moins de 1 ha et 15,50\% plus de 4 ha (Figure 3). La moyenne d'âge des plantations est de 19,88 $\pm 16,65$ ans. Les parcelles sont majoritairement $(49,61 \%)$ représentées par les parcelles en phase de production active (5 à 15 ans). Les plantations matures (15 à 30 ans) et les vieilles plantations (plus de 30 ans) représentent respectivement $29,46 \%$ et $19,38 \%$. Seulement $1,55 \%$ des parcelles sont âgées de moins 5 ans. La plupart des producteurs $(92,25 \%)$ plantent du matériel végétal non amélioré (« tout venant »). Seulement 7,75\% plantent le «cacao Mercedes », matériel végétal sélectionné par le Centre National de Recherche Agronomique (CNRA) en Côte d'Ivoire.

\section{Bioagresseurs des exploitations}

Les producteurs ont mentionné six principaux bioagresseurs responsables des dégâts majeurs dans leurs plantations (Figure 4).

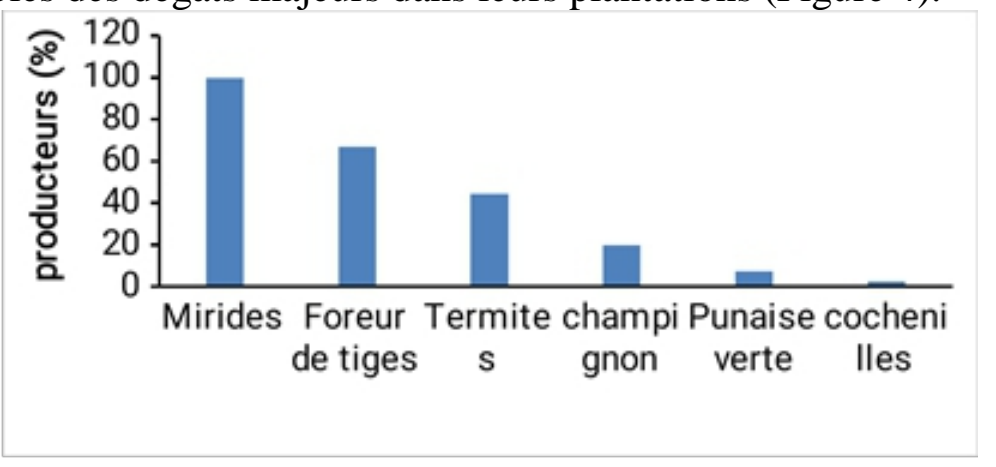

Figure 4. Principaux bioagresseurs cités par les producteurs de cacao

Trois insectes ont été cités comme les plus préoccupants : les mirides Sahlbergella singularis (Haglund), dont les larves et les adultes s'attaquent aux fruits et aux rameaux du cacaoyer; les foreurs de tiges Eulophonotus myrmeleon (Felder), dont les larves creusent des galeries profondes dans le tronc et les branches du cacaoyer et les termites (Isoptère) Ancistrotermes et Microtermes qui s'attaquent aux racines et au tronc du cacaoyer.

Les champignons du genre phytophthora et les Cochenilles farineuses de la famille des Pseudococcidae sont respectivement responsables des maladies de la pourriture brune des cabosses et le Swollen shoot, mais a des taux d'attaques moins préoccupants.

\section{Produits utilisés}

La majorité des enquêtés $(79,84 \%)$ a recours aux produits chimiques pour contrôler ces bioagresseurs. Nous avons relevé le nom de 27 produits phytosanitaires utilisés par les producteurs. Ces produits sont constitués de 24 insecticides et de 3 fongicides. 


\section{Les insecticides}

La majorité des insecticides répertoriés sont homologués et commercialisés par les firmes contre les ravageurs du cacaoyer. Toutefois, deux d'entre eux (2) (Margouza super 50 EC et Leboya 50 EC) ne sont pas homologués. Deux des insecticides (Duel 186 EC et Baythroïde P 168 EC) sont homologués et commercialisés contre les ravageurs du cotonnier. Les insecticides utilisés sont modérément (II) ou légèrement (III) dangereux. Ils appartiennent exclusivement à la famille des néonicotinoïdes, des pyréthrinoïdes et des organophosphorés. Les néonicotinoïdes et les pyréthrinoïdes ont été les plus représentées et sont utilisés essentiellement contre les mirides et contre les foreurs de tiges. Aucun insecticide n'est utilisé contre les termites et les cochenilles responsables du Swollen shoot bien que des attaques et dégâts par ces ravageurs sont mentionnés (Tableau 1).

Tableau 3. Liste des insecticides inventoriés et leur fréquence d'utilisation contre les insectes et maladies les plus dommageables par leurs dégâts.

\begin{tabular}{|c|c|c|c|c|c|c|}
\hline Noms commercial & Familles chimiques & Mirides & $\begin{array}{l}\text { Foreurs } \\
\text { de tiges }\end{array}$ & Termites & S. shoot & $\begin{array}{l}\text { Classe } \\
\text { OMS }\end{array}$ \\
\hline Actara $240 \mathrm{SC}$ & Néonicotinoïde & ++ & - & - & - & III \\
\hline Decis $12,5 \mathrm{EC}$ & Pyréthrinoïde & + & + & - & - & III \\
\hline Caofort $30 \mathrm{SL}$ & Néonicotinoïde & + & - & - & - & II \\
\hline Imidor $200 \mathrm{SL}$ & Néonicotinoïde & + & - & - & - & III \\
\hline Thiosulfan $60 \mathrm{EC}$ & Néonicotinoïde & ++ & - & - & - & III \\
\hline Agricao $45 \mathrm{SC}$ & Néonicotinoïde + Pyréthrinoïde & + & - & - & - & III \\
\hline Boradyne super $45 \mathrm{ZC}$ & Néonicotinoïde + Pyréthrinoïde & + & + & - & - & III \\
\hline Phytacao 45 SC & Néonicotinoïde + Pyréthrinoïde & +++ & - & - & - & III \\
\hline Tropidine $45 \mathrm{SC}$ & Néonicotinoïde + Pyréthrinoïde & + & - & - & - & III \\
\hline Biocao $50 \mathrm{SC}$ & Néonicotinoïde + Pyréthrinoïde & + & - & - & - & III \\
\hline Grosudine super $50 \mathrm{SC}$ & Néonicotinoïde + Pyréthrinoïde & +++ & - & - & - & III \\
\hline Thodan super 35 SC & Néonicotinoïde + Pyréthrinoïde & + & - & - & - & II \\
\hline Protector plus A $45 \mathrm{EC}$ & Néonicotinoïde + Pyréthrinoïde & + & - & - & - & II \\
\hline Cao-plus $25 \mathrm{SC}$ & Néonicotinoïde + Pyréthrinoïde & + & - & - & - & III \\
\hline Onex super $40 \mathrm{SC}$ & Néonicotinoïde + Pyréthrinoïde & + & - & - & - & II \\
\hline Tonnere $88 \mathrm{EC}$ & Néonicotinoïde + Pyréthrinoïde & + & - & - & - & III \\
\hline Duel 186 EC & Organophosphoré + Pyréthrinoïde & - & + & - & - & II \\
\hline Azudines $50 \mathrm{SC}$ & Néonicotinoïde + Pyréthrinoïde & + & - & - & - & II \\
\hline Baythroïde P 168 EC & Organophosphoré + Pyréthrinoïde & - & + & - & - & II \\
\hline Nomax $150 \mathrm{SC}$ & Benzylurées + Pyréthrinoïde & + & - & - & - & III \\
\hline Toro $40 \mathrm{EC}$ & Néonicotinoïde + Pyréthrinoïde & + & - & - & - & II \\
\hline Volcao $40 \mathrm{EC}$ & Néonicotinoïde + Pyréthrinoïde & ++ & - & - & - & III \\
\hline Margouza super 50 EC & Mélange inconnu & + & - & - & - & Obsolète \\
\hline Leboya 50 EC & Mélange inconnu & + & - & - & - & Obsolète \\
\hline
\end{tabular}

Fréquence d'utilisation : +++ (par plus de 10 producteurs), ++ (entre 5 et 10 producteurs), + (entre 1 et 5 producteurs), - (aucuns producteurs) ; II : modérément dangereux ; III : légèrement dangereux 
Près de 70,83\% des insecticides recensés sont constitués de deux matières actives, les autres ne contiennent qu'une seule matière active. Les matières actives les plus fréquemment utilisées sont le Thiaméthoxam et le Lambdacyhalothrine (Figure 5).

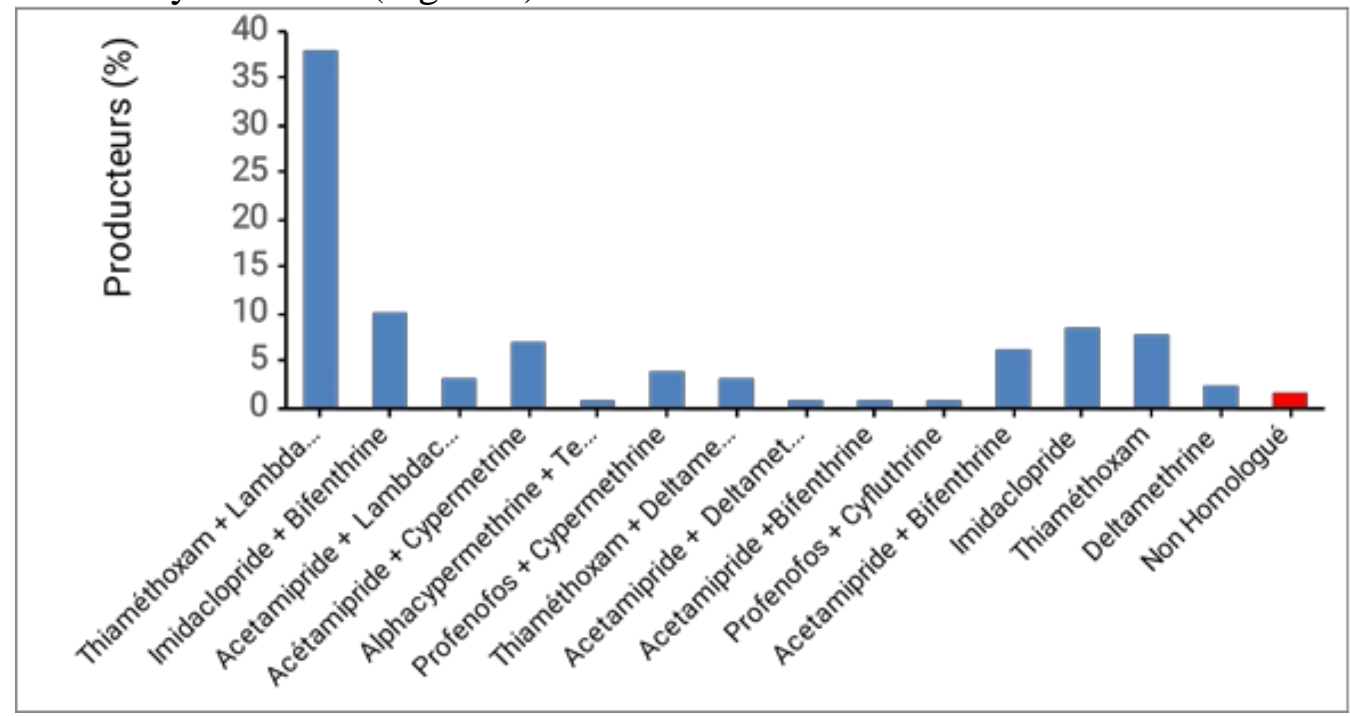

Figure 5. Fréquence d'utilisation des matières actives insecticides

\section{Les fongicides}

Tous les trois fongicides sont homologués et commercialisés par les firmes contre la pourriture brune des cabosses. Ils sont légèrement dangereux (III). Les fongicides appartenant aux familles chimiques des carbamates et des acétamides ont été les plus cités (tableau 2).

Tableau 2. Liste des fongicides répertoriés auprès des producteurs et leur fréquence d'utilisation contre la pourriture brune

\section{Noms commerciaux}

Familles chimiques

\section{Fréquence} d'utilisation

\begin{tabular}{llll}
\hline Forum 46 WP & Acide Cinnamique & + & III \\
Fongicao plus 72 WP & Carbamate + Acétamides & +++ & III \\
Callomil super 66 WP & Amides & + & III \\
\hline
\end{tabular}

Fréquence d'utilisation : +++ (par plus de 10 producteurs), ++ (entre 5 et 10 producteurs $),+$ (entre 1 et 5 producteurs), - (aucuns producteurs) ; III : légèrement dangereux.

Ces fongicides sont tous constitués de deux matières actives. Les matières actives les plus fréquemment utilisées sont le mancozèbe et le cymoxanil (Figure 6). 


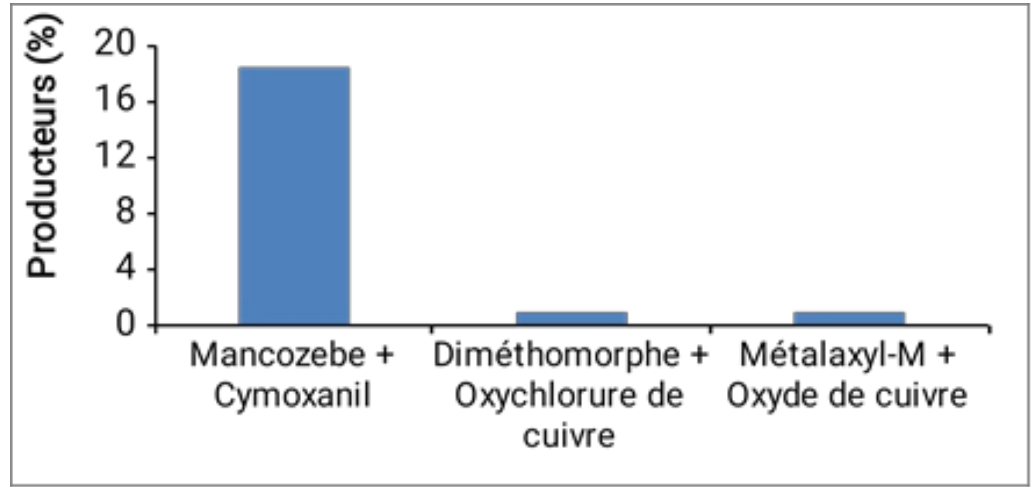

Figure 6. Matières actives fongicides utilisées dans la zone d'étude

\section{Origines}

Nos résultats montrent que la majorité des producteurs $(40,91 \%)$ interrogés achètent leurs produits sur le marché. Trente-cinq virgule quarantecinq pour cent ont déclaré avoir reçu leurs produits des services de l'agriculture. Le reste des enquêtés s'approvisionnent à crédit chez des revendeurs ambulants («pisteurs ») $(13,64 \%)$ ou obtiennent leurs produits auprès des coopératives $(10 \%)$.

\section{Application des produits}

Les producteurs traitent leurs parcelles sans observer le seuil des dégâts des ravageurs. Les traitements sont effectués sans prise en considération des insectes utiles ou du voisinage. La moyenne annuelle de traitement des parcelles est de 2 fois. Vingt-huit virgule seize pour cent les traitent qu'une fois tandis que 1,94\% les traitent jusqu'à 5 fois par an (Figure 7). Les traitements sont majoritairement effectués aux mois de Juillet et d'Août (Figure 8). Ils sont réalisés en majorité $(95,15 \%)$ par des applicateurs professionnels formés par les firmes locales. Ces derniers utilisent des atomiseurs. Près de 4,85\% des producteurs ont déclaré utiliser des pulvérisateurs à dos à pression manuelle pour traiter eux même leurs plantations avec la tenue quotidienne de travail.

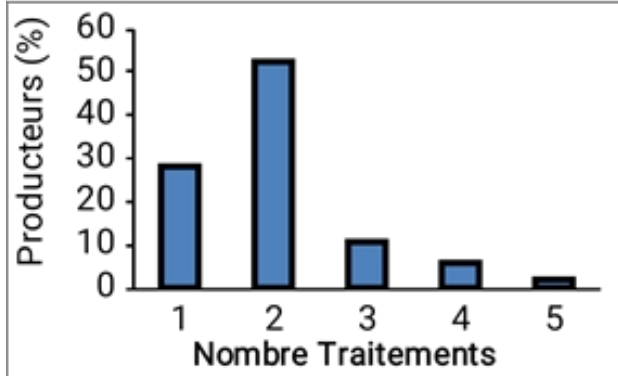

Figure 7 : Nombre annuel de traitements des parcelles

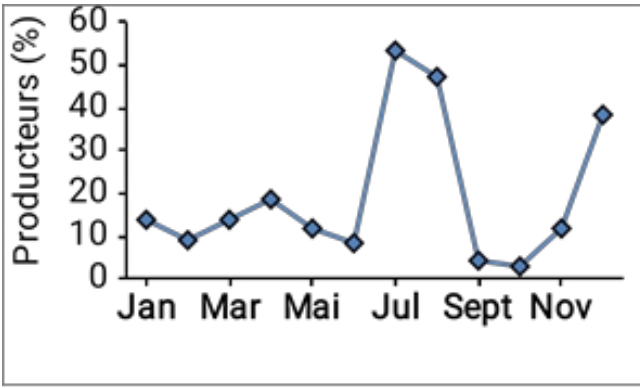

Figure 8 Périodes de traitements des parcelles 
Les traitements sont effectués tôt le matin entre 6 et 10 heures.

\section{Stockage et devenir des emballages vides}

Les producteurs ne disposent pas de magasins de stockage de produits phytosanitaires ; les produits sont stockés dans les habitations $(53,4 \%)$ ou sur les parcelles $(28,16 \%)$ et sont facilement accessibles à tous. Seulement $18,44 \%$ des producteurs les entreposent dans des magasins spéciaux aménagés dans la maison.

Près de $96 \%$ des producteurs abandonnent les emballages vides dans la nature, tandis que $4 \%$ les réutilisent pour conserver le sel de cuisines, les poudres de légumes sèches ou comme une gourde d'eau. Parmi les emballages abandonnés dans la nature, 58,57\% sont laissés sur les parcelles, 4\% loin des parcelles et $33,43 \%$ sont enterrés sur les parcelles.

\section{Discussion}

Les producteurs de cacao de la zone de Daloa sont en majorité $(80,61 \%)$ âgés de plus de 35 ans et généralement $(66,67 \%)$ alphabètes. Ce taux élevé de personnes alphabètes pourrait s'expliquer par un retour au village des jeunes déscolarisés soit à la suite du décès du père soit par le manque de travail dans les villes. Il faudrait également ajouter que dans ces dernières années, des fonctionnaires et certains retraités investissent dans l'agriculture (Assiri et al., 2009).

La plupart $(54,14 \%)$ des producteurs ont des plantations de moins de 2 ha. Ces petites surfaces seraient liées au partage de la plantation familiale parmi les membres de la famille après le décès du chef de famille. La diversification des cultures par les agriculteurs (Ruf \& Schroth, 2013) et le ralentissement ou l'arrêt de la dynamique d'extension cacaoyère face à l'épuisement des réserves forestières du pays (Ruf, 2000; Ruf \& Allangba, 2001) contribueraient aussi au développement de petites parcelles en cacaoculture.

Les plantations ont en majorité entre 5 à 15 ans. Elles seraient en partie le reflet de la régénération ou de la réhabilitation des vielles cacaoyers. La régénération ou la réhabilitation des vieilles cacaoyères serait la meilleure stratégie pour assurer une production durable du cacao ivoirien face au vieillissement des vergers (Assiri et al., 2016).

Les plantations ont été créés exclusivement avec du matériel végétal «tout venant». L'abondance de la variété non améliorée dans les exploitations met en évidence une faible adoption par les producteurs du matériel végétal sélectionné. Ce faible niveau d'adoption des innovations pourrait être lié en partie au faible niveau de revenu des producteurs, ainsi qu'au manque d'information (Assiri et al., 2009). En effet, le coût des 
semences dans la zone d'étude est de $10000 \mathrm{FCFA} / \mathrm{ha}$ pour le « cacao Mercedes » alors que le producteur ne paye rien pour le « tout venant ».

Les mirides, les foreurs de tiges, les termites, le champignon du genre Phytophthora et les cochenilles farineuses ont été cités comme préoccupants. Ce constat de l'importance économique des dégâts de ces bioagresseurs confirme les diagnostics antérieurs. En effet, les mirides piquent et sucent les différents organes du cacaoyer entrainant des pertes de près de $30 \%$ en Côte d'Ivoire (Lavarbe, 1977). Les foreurs de tiges Eulophonotus myrmeleon perforent les tiges du cacaoyer provoquant près de $25,8 \%$ de perte en Côte d'ivoire (N'Guessan et al., 2014). Le champignon du genre Phytophthora attaque les cabosses du cacaoyer provoquant des taux d'attaques de plus de $40 \%$ en absence de traitements en plantation villageoise (Pohe et al., 2013). Les termites s'attaquent aussi aux pieds des cacaoyers et peuvent provoquer la mort des plants ou la mort totale des vergers en Côte d'Ivoire (Tra Bi et al., 2010).

Seulement $79,84 \%$ des producteurs ont déclaré effectuer des traitements phytosanitaires. Les pesticides utilisés sont majoritairement les insecticides. L'usage des pesticides serait fortement corrélé aux types de ravageurs et aux taux d'attaque. La lutte chimique contre les termites est presqu'inexistante dans notre zone d'étude. La lutte mécanique est la plus pratiquée par les producteurs pour détruire les nids et les placages de termites sur les pieds des cacaoyers. Cependant, pour que ce procédé mécanique ait un niveau d'efficacité, il faudrait absolument s'assurer que la reine a été atteinte et détruite. Les néonicotinoïdes, les pyréthrinoïdes et les organophosphorés ont été les plus représentés et utilisés contre les mirides et les foreurs de tiges. Le choix de ces familles chimiques pourrait s'expliquer par leur efficacité (Sparks, 2013). Ces familles chimiques dominent aussi largement le marché mondial des insecticides (Jeschke et al., 2011).

Nos résultats ont révélé l'utilisation de deux insecticides normalement homologués pour le control du ravageur du cotonnier Bemisia tabaci (Hémiptères). Bellec et al. (2017) ont observé des cas similaires chez les producteurs de légumes mauriciens qui utiliseraient l'hexaconazole, fongicide autrefois utilisé pour lutter contre les maladies du blé et de l'orge. Par ailleurs, deux insecticides, le Margouza super 50 EC et Leboya 50 EC sont utilisés contre les mirides bien qu'ils soient non homologués en Côte d'Ivoire.

Bien que ces produits soient modérément ou légèrement toxiques, leur utilisation prolongée peut entraîner une toxicité chronique ou des impacts négatifs sur l'environnement. En effet, le contact de l'homme avec de nombreux composés chimiques au cours de sa vie pourrait entraîner le développement de certains cancers, l'apparition d'allergies, de troubles neurologiques ou encore la perturbation de la reproduction (Mnif et al., 2011). 
Les pyréthrinoïdes auraient une toxicité aigüe et chronique létale élevée (Claver, 2005) car la deltaméthrine et la lambdacyhalothrine, fréquemment utilisées, pourraient provoquer des perturbations endocrines en affectant le système hormonal de l'organisme, ce qui pourrait causer aussi une augmentation des malformations congénitales, des anomalies sexuelles et des défaillances reproductives ; la cyperméthrine, pourrait aussi être cancérigène pour l'homme (Alavanja et al., 2003; Lee et al., 2004). L'exposition aux organophosphorés pourrait affecter la qualité du spermatozoïde (Perry et al., 2011). Les carbamates seraient plus toxiques à l'égard des invertébrés et des poissons (Eddaya et al., 2015).

Les recommandations sur les traitements phytosanitaires sont peu respectées. Les résultats obtenus montrent que les interventions phytosanitaires sont insuffisantes. En effet, il est apparu que la majorité des producteurs $(52,43 \%)$ traitent deux fois leur plantation sur les quatre qui sont recommandées par an (Anonymous, 2005). Les périodes de traitements par contre sont mieux respectées. En effet, les résultats obtenus montrent 53,4\% des producteurs respectent les périodes et les délais préconisées (Anonymous, 2005).

S'agissant de l'origine des produits, nos résultats ont montré que la majorité des producteurs s'approvisionnaient sur les marchés locaux ou préfèrent prendre à crédit chez des revendeurs ambulants («pisteurs »). Les deux insecticides non homologués (Margouza super 50 EC et Leboya 50 EC) sont vendus par les revendeurs ambulants. Les prix de vente sont inférieurs à ceux offerts au niveau des canaux officiels tels que les magasins de distribution agréent.

Quelques producteurs $(6,8 \%)$ effectuent leur traitement avec leur tenue quotidienne de travail, constituée de vieux habits. Ils utilisent des pulvérisateurs à dos à pression manuelle. L'utilisation de pulvérisateurs à dos à pression manuelle n'est pas exempte de risques en raison des fuites fréquentes de liquide observées, d'une mauvaise protection des producteurs et du vieillissement des équipements mal entretenus (Ntow et al., 2006 ; Matthews et $a l .$, 2009). Ce fait pourrait s'expliquer par un faible niveau des revenus des producteurs qui ne peuvent ni payer les applicateurs professionnels ni acheter les combinaisons de protection.

Nos résultats ont révélé également que la majorité des producteurs $(53,4 \%)$ stockent les produits dans les habitations à accès libre. Cela pourrait être l'origine d'intoxications surtout chez les enfants. En effet, dans la localité de Yamoussoukro (Côte d'Ivoire) un décès et 10 intoxications d'enfants ont été signalés suite à la consommation de poissons contaminés par des pesticides (Anonymous, 2009).

En ce qui concerne les emballages vides, 96\% des producteurs les abandonnent dans la nature, tandis que $4 \%$ les réutilisent pour les activités 
culinaires ou comme gourde d'eau pour les activités champêtres. La gestion rationnelle des emballages vides est quasi inexistante dans la zone d'étude. La réutilisation d'emballages vides des pesticides présenterait des risques. En effets, même lorsque les emballages semblent propres, il reste toujours des résidus de produits à l'intérieur, absorbés dans les parois de l'emballage, ce qui fait de ces derniers des déchets spéciaux (Schiffers \& Mar, 2011). C'est ainsi que six personnes intoxiquées dans la localité de Korhogo après avoir consommé de la nourriture préparée en utilisant un emballage vide de pesticides sont décédées (Anonymous, 2018). Autrement dit, les emballages abandonnés dans la nature peuvent être nocifs aussi bien à 1'Homme qu'à l'environnement (Samuel \& Saint Laurent, 2001).

\section{Conclusion}

La majorité des producteurs utilisent des pesticides homologués et commercialisés par les firmes contre les mirides, les foreurs de tiges ou les champignons du genre Phytophthora, responsables de la pourriture brune des cabosses et d'autres maladies. La lutte chimique contre les termites a été quasi inexistante dans notre zone d'étude bien que les dégâts par des termites sont cités comme majeurs. Toutefois, la méthode mécanique de lutte contre ces derniers est utilisée. Des détournements d'usage de pesticides (application d'un pesticide sur une culture sur laquelle il n'est pas homologué) et l'usage de pesticides non homologués ont été aussi observés. Les pesticides utilisés ne sont pas stockés dans les conditions adaptées. De plus, la gestion rationnelle des emballages vides est quasi inexistante. Ces usages seraient symptomatiques d'un manque de connaissance des utilisateurs des effets néfastes de ces pesticides.

\section{References:}

1. Alavanja, M.C.R., Samanic, C., Dosemecil, M., Lubin, J., Tarone, R., Lynch, C.F., Knott, C., Thomas, K., Hoppin, J.A., Barker, J., Coble, J., Sandler, D.P., \& Blair, A. (2003). Use of Agricultural pesticides and prostate cancer Risk in agricultural health study cohort. Am. J. Epidemiol., 157 :800 - 814.

2. Anonyme (2005). Bien cultiver le cacaoyer en Côte d'Ivoire. Fiche Technique. Centre National de Recherche Agronomique (CNRA) : 4

3. Anonyme (2009). Yamoussoukro, intoxication alimentaire à Gourominakro: un enfant mort et 9 miraculés. DOI : www.koffi.net/koffi/actualité/58754-yamoussoukro-intox. Consulté le 25 mai 2018.

4. Anonyme (2018). Les populations de Nandalla appelées à se débarrasser des emballages de pesticides après la dramatique 
intoxication alimentaire. DOI : https/news.abidjan.net/h/635835.html. Consulté le 25 mai 2018.

5. Anonyme (2018). Climat : Haut-sassandra. DIO : http://fr.climatedata.org/region/1313/. Consulté le 01 septembre 2018.

6. Assiri, A.A., Deheuvels, O., Keli, Z.J., Kebe, B.I., Konan, A., \& Koffi, N. (2016). Identification des caractéristiques agronomiques pour le diagnostic et la prise de décision de régénération des vergers de cacaoyers en côte d'ivoire. Afr. Crop Scie. J., 24 (3) : 223 -234.

7. Assiri, A.A., Yoro, G.R., Deheuvels, O., Kébé, B.I., Kéli, Z.J., Adiko, A., \& Assa, A. (2009). Les caractéristiques agronomiques des vergers de cacaoyer (Theobroma cacao L.) en Côte d'Ivoire. J. Anim. Plant Sci., 2 (1) : 55 - 66.

8. Brou, Y.T., Akindès, F., \& Bigot, S. (2005). La variabilité climatique en Côte d'Ivoire: entre perceptions sociales et réponses agricoles. Cah. Agric., 5(6) : 533 - 540.

9. Clavet, R., Barriuso, E., Benoit, P., Charnay, M.P., \& Coquet, Y. (2005). Les pesticides dans le sol: conséquences agronomiques et environnementales, France agricole. Ed.

10. Eddaya, T., Boughdat, A., becker, L., Chaimbault, P., \& Zaïd, A. (2015). Utilisation et risques des pesticides en protection sanitaire de la menthe verte dans le Centre - Sud du Maroc. J. mater Environ. Sci., $6(3): 656-665$.

11. Hoyer, A.P., Gerdes, A.M., Jorgensen, T., Rank, F., \& Hartvig, H.B. (2002). Organochlorines, p53 mutations in relation tobreast cancer risk and survival. A Danish cohort-nested case-controls study. Breast Cancer Res. Treat., 71 (1) : 59 - 65.

12. Jescke, P., Nauen, R., Schindler, M., \& Elbert, A. (2011). Overview of the Status and Global Strategy for Neonicotinoids, J. Agric. Food Chem., $59: 2897-2908$.

13. Kouakou, K., Kébé, B.I., Kouassi, N., Ann, A.P., Aké, S., \& Muller, E. (2011). Impact de la maladie virale Swollen shoot du cacaoyer sur la production de cacao en milieu paysan à Bazré (Côte d'Ivoire). $J$. Appl. Biosci., 43 : 2947 - 2957.

14. Kouamé, N.N., N'Guessan, K.F., N'Guessan, H.A., N'Guessan, P.W., \& Tano, Y. (2015). Variations saisonnières des populations de mirides du cacaoyer dans la région du Haut Sassandra en Côte d'Ivoire. J Anim Plant Sci., 25(1) : 3787-3798.

15. Lajide, L., Escoubas, P., \& Mitzutani, J. (1995). Termite antifeedant activity in Xylopia aethiopica. Phytochemistry., 40 (4) : 1105-1112.

16. Lavabre, E. (1977). Importance économique des mirides dans la cacaoculture mondiale. In. Les mirides du cacaoyer. Institut Français du café et du cacao., 155 - 170. 
17. Le Bellec, F., Maud Scorbiac, M., \& Sauzier, J. (2017). Les pratiques phytosanitaires des producteurs de légumes de l'île Maurice : impacts et perspectives de changement. Cah. Agric., $26: 55001$.

18. Lee, W.J., Blair, A., Hoppin, J.A., Lubin, J.H., Rusiecki, J.A., Sandler, D.P., Dosemeci, M., \& Alavanja, M.C.R. (2004). Cancer incidence among pesticides applicators exposed to chlorpyrifos in the agricultural health study. J. Natl. Cancer Inst. 96 (23): 1781 - 1789.

19. Matthews, G., Wiles, T., \& Baleguel, P. (2003). A survey of pesticides application in Cameroon, Crop. Prot., $22: 707-714$.

20. Mnif, W., Hassine, A.I.H., Bouaziz, A., Bartegi, A., Thomas, O., \& Roig, B. (2011). Effect of endocrine disruptor pesticides. Int. J. Environ. Res. Public Health., 8: 2265 -2303.

21. N'Guessan, H.A., N'Guessan, K.F., Kouassi, K.P., Kouamé, N.N., \& N'Guessan, P.W. (2014). Dynamique des populations du foreur de tiges du cacaoyer, Eulophonotus mymeleon Felder (Lepidoptère: Cossidae) dans la région du Haut- Sassandra en Côte d'Ivoire. J. appl. Biosci., 83 :7606 -7614.

22. Ntow, W.J., Gijzen, H.J., Kelderman, P., \& Drechsel, P. (2006). Farmer percetions and pesticide use practices in vegetable production in Ghana. Pest manag. Sci., $62: 356-365$.

23. Perry, M.J., Venners, S.A., Chen, X., Liu, X., Tang, G., Xing, H., Barr, D.B., \& Xu, X. (2011). Organophosphorous pesticide exposures and sperm quality Reproductive Toxicologie., 31(1):75 - 79.

24. Pohe, J., Pohe, S., Steve, W., \& Okou, S.F.F. (2013). L'huile des graines de neem, un fongicide alternatif à l'oxyde de cuivre dans la lutte contre la pourriture brune des cabosses de cacaoyer en Côte d'Ivoire. J. appl. Biosci., 16 (3) : 2362 - 2368.

25. Ruf, F. (2000). Déterminants sociaux et économiques de la replantation. Oléagineux, Corps Gras, Lipides., 7 (2) : 189 -196.

26. Ruf, F. \& Allangba, K. (2001). Décisions de plantation et replantation cacaoyères. Le cas des migrants Baoulés à Oumé (Côte d'Ivoire). In : R.Y Assamoi, K. Burger, D Nicolas, F. Ruf et P. de Vernou, eds. $L$ 'avenir des cultures pérennes. 5-9 novembre 2001. Yamoussoukro (Côte d'Ivoire) : BNETD \& CIRAD.

27. Ruf, F. \& Schroth, G. (2013). Économie et écologie de la diversification des cultures pérennes tropicales. In : Cultures pérennes tropicales : enjeux économiques et écologiques de la diversification. Versailles : Éditions Quae: 5-30. DIO : http://excerpts.numilog. com/books/9782759218547.pdf.

28. Samuel, O. \& Saint-Laurent, L. (2001). Guide de prévention pour les utilisateurs de pesticides en agriculture maraîchère. Institut de Recherche en Santé, Quebec, 92. 
29. Schiffers, B. \& Mar, A. (2011). Sécurité des opérateurs et bonnes pratiques phytosanitaires. Manuel 4. Pip c/o Coleacp. Bruxelles, Belgique, mars 2011. DIO : www.coleacp.org/pip.

30. Sparks, T.C. (2013). Insecticide discovery: An evaluation and analysis. Pest. Biochem. Physiol., $107: 8$ - 17.

31. Tra-Bi, C.S., Konaté, S., \& Tano, Y. (2010). Diversité et abondance des termites (Insecta : Isoptera) dans un gradient d'âges de paillis de cabosses (Oumé - Côte d'ivoire). J. anim. plant sci., 6(3) : 685 - 699. 